\title{
B3GnT8 regulates the metastatic potential of colorectal carcinoma cells by altering the glycosylation of CD147
}

\author{
JIANLONG NI $^{1 *}$, ZHI JIANG $^{1 *}$, LI SHEN ${ }^{1}$, LIPING GAO $^{1}$, MEIYUN YU $^{1}$, \\ $\mathrm{XU} \mathrm{XU}^{1}$, SHITAO ZOU $^{2}$, DONG HUA ${ }^{2}$ and SHILIANG WU ${ }^{1}$ \\ ${ }^{1}$ Department of Biochemistry and Molecular Biology, School of Medicine, Soochow University, Suzhou, \\ Jiangsu 215123; ${ }^{2}$ The Fourth Affiliated Hospital of Soochow University, Wuxi, Jiangsu 214062, P.R. China
}

Received December 5, 2013; Accepted January 28, 2014

DOI: $10.3892 /$ or.2014.3042

\begin{abstract}
Aberrant glycosylation of cell surface glycoproteins is commonly associated with the invasion and metastasis of colorectal carcinomas, which can be attributed to the upregulated expression of glycosyltransferases. Therefore, elucidation of glycosyltransferases and their substrates may improve our understanding of their roles in tumor metastasis. $\beta-1,3-\mathrm{N}-$ acetylglucosaminyltransferase- 8 ( $\beta 3 \mathrm{GnT} 8)$ is a key enzyme that catalyzes the formation of poly- $\mathrm{N}$-acetyllactosamine (polylactosamine) chains on $\beta 1,6$-branched $\mathrm{N}$-glycans in vitro, which is also involved in tumor invasion. In the present study, we analyzed the expression of $\beta 3 \mathrm{GnT} 8$ and its product polylactosamine in four human colorectal carcinoma cell lines (LS-174T, SW620, SW480 and LoVo) with different metastatic potential. We found that the levels of $\beta 3 \mathrm{GnT} 8$ and polylactosamine chains were gradually increased in the colorectal cancer cell lines in a trend from low to high metastatic potential. Notably, a significantly positive relationship between $\beta 3 \mathrm{GnT} 8$ expression and HG-CD147 was noted in the colorectal cancer cell lines. To further investigate their relationships, exogenous $\beta 3 \mathrm{GnT} 8$ was introduced into the LS-174T cells, while expression of $\beta 3 \mathrm{GnT} 8$ was downregulated in the LoVo cells. The overexpression of $\beta 3 \mathrm{GnT} 8$ in LS-174T cells increased the level of HG-CD147. Conversely, downregulation of $\beta 3 \mathrm{GnT} 8$ expression in LoVo cells significantly decreased the expression of HG-CD147. HG-CD147 is a major carrier of $\beta 1,6$-branched polylactosamine sugars; therefore, the regulation of $\beta 3 \mathrm{GnT} 8$ significantly altered the $\beta 1,6$-branched polylactosamine struc-
\end{abstract}

Correspondence to: Dr Shiliang Wu, Department of Biochemistry and Molecular Biology, School of Medicine, Soochow University, Suzhou, Jiangsu 215123, P.R. China

E-mail: shiliang_wu@126.com

Dr Dong Hua, The Fourth Affiliated Hospital of Soochow University, Wuxi, Jiangsu 214062, P.R. China

E-mail: huadong@csco.org.cn

*Contributed equally

Key words: $\beta 3 \mathrm{GnT} 8$, polylactosamine, colorectal carcinoma cells, HG-CD147, metastasis tures on CD147. Hence, we suggest that $\beta 3 \mathrm{GnT} 8$ plays a key role in the metastasis of colorectal cancer cells by altering the $\beta 1,6$-branched polylactosamine sugars of CD147.

\section{Introduction}

Glycosylation products of proteins are ubiquitous components of the cell membrane, and glycosylated molecules are involved in a broad variety of biological functions related to cell-cell communication and cellular behaviors (1). Furthermore, aberrant glycosylation of cell surface glycoproteins due to specific alterations of glycosyltransferases is associated with carcinogenesis and plays an importment role in cancer invasion and metastasis (2). For example, comparison of N-glycans from different colon cancer cell lines revealed that highly metastatic cancer cells contained more polylactosamine side chains when compared with cells with low metastatic potential (3).

Colorectal cancer is one of the most common types of malignancy. Approximately half of colorectal cancer patients develop metastatic lesions and present with poor prognosis (4). The mechanisms of colorectal cancer metastasis are multifaceted and extremely intricate, mainly including the following key steps: cancer cell migration, adhesion, invasion, growth, neovascularization, specific organ homing and immune evasion (5). The increasing body of research on colorectal cancer metastasis indicates that glycosyltransferases affect metastasis by altering glycosylation of glycoproteins associated with metastasis. Ishida et al (6) found that the level of the $\beta 3 \mathrm{GnT} 8$ transcript was significantly higher in colon cancer than that in other cancer types. Our previous research demonstrated that expression of $\beta 3 \mathrm{GnT} 8$ is associated with the invasive potential of the AGS cell line (7).

$\beta 3 \mathrm{GnT} 8$ is a member of the $\beta 3 \mathrm{GnT}$ family, which is involved in the biosynthesis of polylactosamine chains on $\beta 1,6$-branched $\mathrm{N}$-glycans in vitro (6). It transfers GlcNAc to the non-reducing terminus of the Gal $\beta 1-4 \mathrm{GlcNAc}$ of tetra-antennary N-glycan to form polylactosamine structures in vitro. Polylactosamine comprises repeated (Gal $\beta 1-4 \mathrm{GlcNAc} \beta 1-3) n$, and attaches to $\mathrm{O}$-glycans, N-glycans or glycolipids. It has been shown that highly metastatic carcinoma cells express more polylactosamine chains than cells with low metastatic potential (3).

CD147, a target glycoprotein of $\beta 3 \mathrm{GnT} 8$, which is also known as extracellular matrix metalloproteinase inducer 
(EMMPRIN) is widely expressed at high levels on human tumor cells $(8,9)$. Due to the heterogeneous N-glycosylation, CD147 shows both high glycosylated (HG)-CD147 ( 40-60 kDa) and low glycosylated (LG)-CD147 ( 32 kDa) forms. A significant biochemical property of CD147 is its high level of glycosylation. The extracellular region of CD147 contains three N-linked glycosylation sites (Asn44, Asn152 and Asn186), and N-glycosylation contributes to almost half the size of the mature CD147 molecule (10). It is HG-CD147 that is active in the induction of MMPs thereby leading to extracellular matrix degradation and increased tumor growth and metastasis (11).

In the present study, four human colorectal cancer cell lines, LS-174T, SW480, SW620 and LoVo, of different metastatic potential were chosen to study the relationship between colorectal cancer metastatic ability and the expression levels of $\beta 3 \mathrm{GnT} 8, \mathrm{CD} 147$ and polylactosamine. First, we investigated their expression levels in the four colorectal cancer cell lines with different metastatic potential, and the invasion and migration ability. In addition, exogenous $\beta 3 \mathrm{GnT} 8$ was introduced into $\beta 3 \mathrm{GnT}$ 8-low LS-174T cells, and the high expression of $\beta 3 \mathrm{GnT} 8$ was downregulated in LoVo cells. We aimed to identify the relationship between the function of $\beta 3 \mathrm{GnT} 8$ and the metastatic potential of colorectal cancer cell lines and to speculate whether $\beta 3 \mathrm{GnT} 8$ expression affects the glycosylated forms of CD147 to further influence the metastasic ability of colorectal cancer.

\section{Materials and methods}

Cell culture. LS-174T, SW480, SW620 and LoVo cells were obtained from the American Type Culture Collection (ATCC; Manassas, VA, USA), and were cultured in RPMI-1640 medium (Gibco-BRL/Life Technologies, Gaithersburg, MD, USA), supplemented with $10 \%$ fetal bovine serum (FBS) in a humidified atmosphere with $5 \% \mathrm{CO}_{2}$ at $37^{\circ} \mathrm{C}$.

Cell transfection. The recombinant plasmid pEGFP-C1$\beta 3 \mathrm{GnT} 8$ and pSilenCircle-Si- $\beta 3 \mathrm{GnT} 8$ were preserved in our laboratory. Cells were seeded in a 6 -well plate at the density of $8 \times 10^{5} / \mathrm{ml}, 2 \mathrm{ml} /$ well. After cell attachment, the two recombinant plasmids were transfected into LS-174T and LoVo cells, respectively, using Lipofectamine ${ }^{\mathrm{TM}}$ 2000. At the same time, cells transfected with the empty vector pEGFP-C1 were used as a mock, and the untransfected control group was also set up. The effects of pEGFP-C1- $\beta 3 \mathrm{GnT} 8$ and pSilenCircle-Si$\beta 3 \mathrm{GnT} 8$ were confirmed by the expression of $\beta 3 \mathrm{GnT} 8$ protein using western blot analysis.

Western blot analysis. Cells were harvested and homogenized with lysis buffer $(150 \mathrm{mM} \mathrm{NaCl}, 10 \mathrm{mM}$ Tris, $\mathrm{pH} \mathrm{7.5,1 \%}$ NP-40, $1 \%$ deoxycholate, $0.1 \%$ SDS, protease inhibitor cocktail) (Roche Applied Science, Indianapolis, IN, USA). Proteins from cells were resolved on 10\% SDS-PAGE gel (Invitrogen, Carlsbad, CA, USA) and transferred to nitrocellulose membranes (Millipore, Bedford, MA, USA). The membranes were blocked with $5 \%$ skim milk or $1 \%$ bovine serum albumin (BSA) in Tris-buffered saline (TBS) buffer $(10 \mathrm{mM}$ Tris, $150 \mathrm{mM} \mathrm{NaCl}, \mathrm{pH}$ 7.9) containing $0.05 \%$ Tween-20. The proteins were analyzed using specific antibodies as indicated, followed by incubation with appropriate primary antibodies at $4^{\circ} \mathrm{C}$ overnight. After washing in TBS Tween-20 buffer, the membranes were incubated for $2 \mathrm{~h}$ with the appropriate peroxidase-conjugated secondary antibodies. After washing in TBS Tween-20 buffer, the protein bands on the membranes were visualized using an ECL kit (GE Healthcare). Rabbit anti-human $\beta 3 \mathrm{GnT} 8$ affinity $\mathrm{pAb}$ was purified in our laboratory. $\beta$-actin and CD147 antibodies were purchased from Santa Cruz Biotechnology (Santa Cruz, CA, USA).

Flow cytometric analysis. To detect polylactosamine structures of cell surface glycoproteins, biotin-labeled LEA (Sigma, St. Louis, MO, USA) which is specific for binding to polylactosamine residues was used. Cells were detached with $0.25 \%$ trypsin-EDTA solution, then washed three times with PBS. The cell density was adjusted to $3 \times 10 \% / \mathrm{ml}$, and cells were stained with $10 \mu \mathrm{g} / \mathrm{ml}$ LEA PBS (containing $0.5 \%$ BSA and $0.05 \%$ sodium azide) at $37^{\circ} \mathrm{C}$ for $1 \mathrm{~h}$, then washed three times with PBST (PBS $+0.05 \%$ Tween-20). Staining was carried out with $10 \mu \mathrm{g} / \mathrm{ml}$ PE (phycoerythrin)-conjugated streptavidin (Sigma) at $37^{\circ} \mathrm{C}$ for $1 \mathrm{~h}$, and the cells were then washed three times with PBST. The fluorescence intensity of the stained cells was measured using a flow cytometer and analyzed with CellQuest software (BD Biosciences, Franklin Lakes, NJ, USA).

Lectin blot analysis. Cells were harvested and lysed, and $10 \mu \mathrm{g}$ of proteins was subjected to SDS/PAGE (10\% gels). After electrophoresis, the gels were blotted onto PVDF membranes. The membranes were incubated with $3 \%$ BSA in TBS (Tris-buffered saline: $20 \mathrm{mM}$ Tris and $0.5 \mathrm{M} \mathrm{NaCl}, \mathrm{pH} 7.5$ ) overnight and then the blots were incubated with biotinylated LEA (1:400 dilution) in PBST for $1 \mathrm{~h}$. After washing with PBST, the membranes were incubated with streptavidin-HRP (1:1,000 dilution) for $1 \mathrm{~h}$ and then washed with PBST. Staining was detected with ECL western blot detection reagents. A PageRuler Prestained Protein Ladder Plus (Fermentas Life Sciences) was used for sizing of proteins on the lectin blots.

Transwell invasion and migration assays. To evaluate cell invasion capability, Transwell plates (Corning Life Sciences, Tewksbury, MA, USA) with a filter diameter of $6.5 \mathrm{~mm}$ and pore size of $8.0 \mu \mathrm{m}$ were used (17). Transwell chambers were inserted into a 24-well plate, and the upper parts of the inserted membranes were coated with Matrigel. Cells were diluted to $5 \times 10^{5}$ cells $/ \mathrm{ml}$ in RIPA-1640 containing $0.5 \%$ BSA, and $5 \times 10^{5}$ cells/well were seeded into the upper compartment. The lower compartment of the chamber was filled with $500 \mu \mathrm{l}$ complete RIPA-1640 medium with $10 \%$ FBS. The chambers were incubated at $37^{\circ} \mathrm{C}$ for $48 \mathrm{~h}$ and then removed from the plate. Cells and Matrigel were removed with cotton swabs, and the filters were fixed in $95 \%$ ethanol for $30 \mathrm{~min}$ and then washed twice in distilled water. Filters were stained with eosin staining solution for $20 \mathrm{~min}$, rinsed twice with distilled water and examined via light microscopy. Cells were counted under a microscope at x200 magnification. Data were obtained from three individual experiments performed in triplicate.

Cell migration assay was similarly performed, except that the upper parts of the inserted membranes were not coated with Matrigel. Cells were incubated at $37^{\circ} \mathrm{C}$ for $24 \mathrm{~h}$. Cells that had migrated to the lower parts of the chamber were stained 

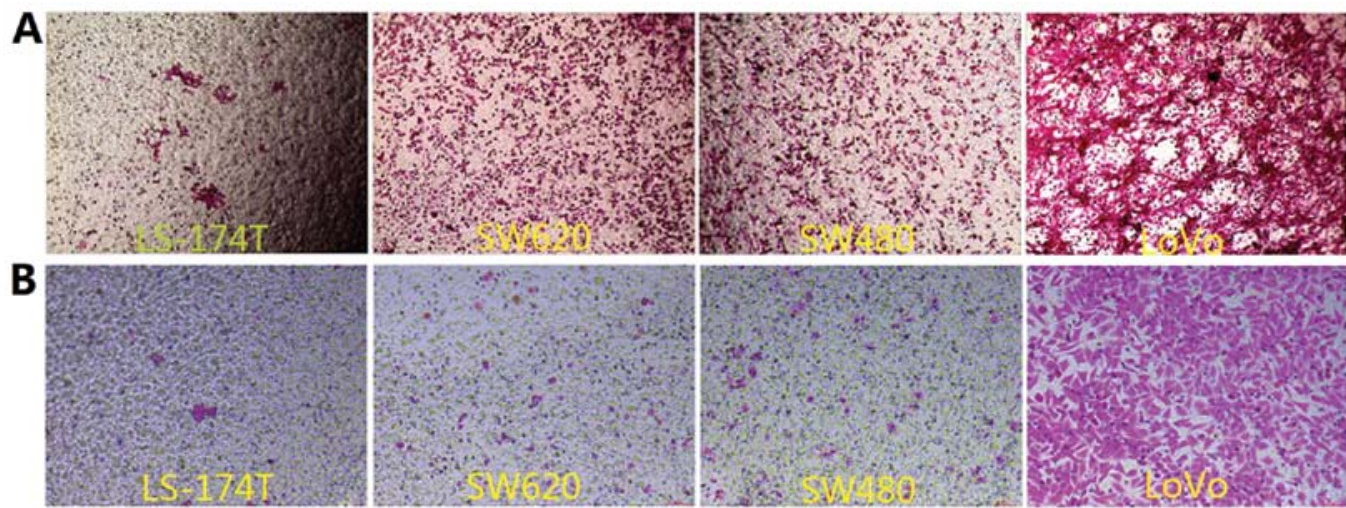

C

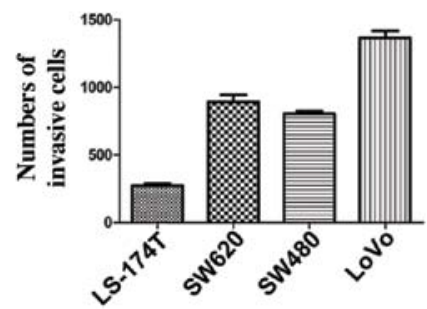

D

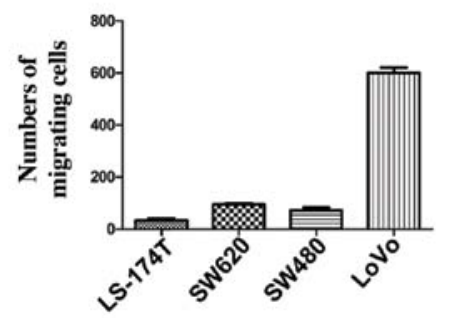

Figure 1. Transwell invasive and migration assays were carried out to compare and quantify the invasive and migratory capabilities of LS-174T, SW620, SW480 and LoVo cells. (A) Transwell invasion assay. Cells were seeded into a Transwell chamber. The cells that passed through the Matrigel-coated polycarbonate membrane were fixed and stained with eosin staining solution and then examined using light microscopy at x200 magnification. (B) Transwell migration assay. Representative examples were photographed at x200 magnification. (C) The relative number of invasive cells. (D) The relative number of migratory cells. Results are representative of three independent experiments, and bars represent the mean \pm SD.

and counted as described above. Data were obtained from three individual experiments performed in triplicate.

Statistical analysis. Statistical analysis was performed using the SPSS13.0 software. Each assay was performed at least three times. Data are expressed as means \pm SD. The Student's t-test was used to evaluate the significance of data. A P-value $<0.05$ was considered to indicate a statistically significant result.

\section{Results}

Transwell invasion and migration assays. To confirm the invasion and migration of the four colorectal cancer cell lines with different metastatic potential, Transwell migration and invasion chambers coated with Matrigel were used. As shown in Fig. 1A and B, the Transwell invasion and migration experiments showed that the number of LoVo cells that passed through the polycarbonate membrane was much higher than the number of LS-174T cells. The numbers of invasive and migratory SW480 and SW620 cells were between the values noted for the LoVo and LS-174T cells. The results confirmed that the invasive and migratory capabilities of the four colorectal cancer cell lines were consistent with their metastatic potentials; LoVo cells had the highest invasive and migratory capabilities, LS-174T cells had the lowest, while SW480 and SW620 cells had moderate capabilities.

Differential expression of $\beta 3 G n T 8$ in the four colorectal cancer cell lines. Proteins from the four colorectal cancer cells were assessed by western blot analysis. As shown in Fig. 2, western blot results showed that $\beta 3 \mathrm{GnT} 8$ protein exhibited differential expression in the four colorectal cancer cell lines.
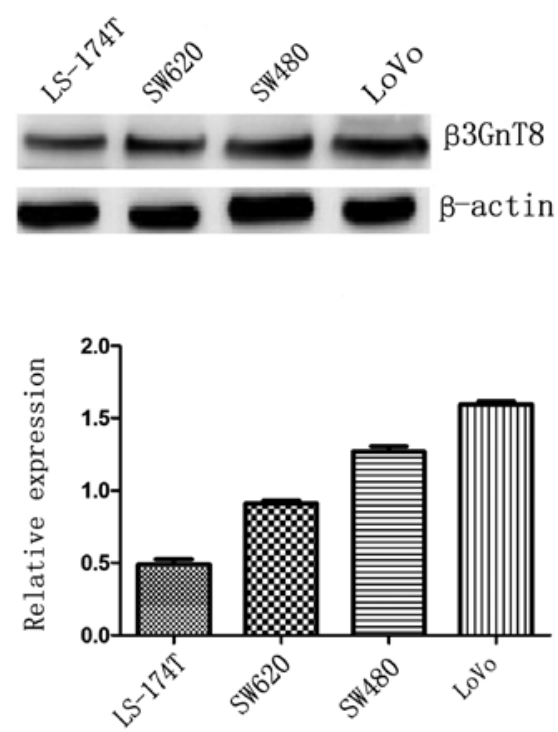

Figure 2. Western blot analysis of $\beta 3 \mathrm{GnT} 8$ expression in the four colorectal cancer cell lines. (A) Blots were stripped and reprobed with a human $\beta$-actin probe to confirm equal loading. In total, $30 \mu \mathrm{g}$ of total protein was loaded in each lane. (B) The relative expression of $\beta 3 \mathrm{GnT} 8$ protein. The experiments are representative of three independent experiments with similar results, and bars represented the mean $\pm \mathrm{SD}$.

The highest level of $\beta 3 \mathrm{GnT} 8$ protein was detected in the LoVo cells. The lowest level was noted in the LS-174T cells, and $\beta 3 \mathrm{GnT} 8$ protein expression in the SW480 and SW620 cells was between the levels noted in the LoVo and LS-174T cells. The results revealed that with the increasing capacity of metastasis of the cell lines, the expression of $\beta 3 \mathrm{GnT} 8$ was also gradually increased. 

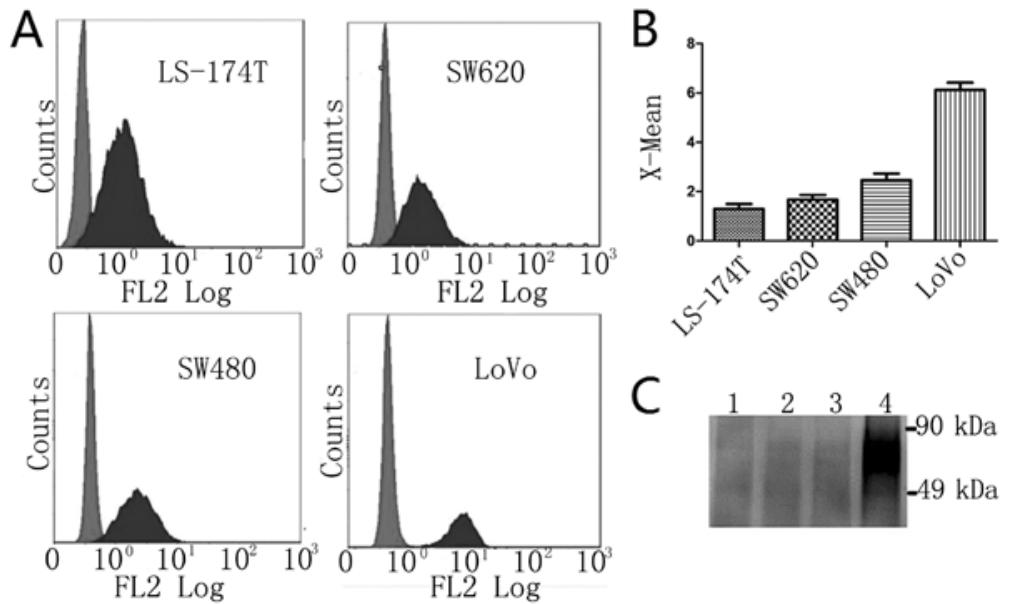

Figure 3. Flow cytometric analysis of polylactosamine residues and the lectin blot method in the four colorectal cancer cell lines. (A) Results of the flow cytometric analysis. The light region represents the control group, and the dark region represents the experimental group. LoVo cells exhibited the highest expression of polylactosamine residues of the four colorectal cancer cell lines. Compared to the LoVo cells, the level of polylactosamine residues in the LS-174T cells was extremely low. (B) The X-mean values of the four colorectal cancer cells by flow cytometric analysis. Results are representative of three independent experiments, and bars represent the mean \pm SD. (C) Polylactosamine residues on glycoproteins using lectin blot analysis. Lanes 1, 2, 3 and 4 represents LS-174T, SW620, SW480 and LoVo cells, respectively.

Level of polylactosamine in the four colorectal cancer cell lines. We subsequently examined the expression levels of polylactosamine on the cell surface of the four colorectal cancer cell lines using flow cytometry and lectin blot method. Polylactosamine residues can be specifically identified by tomato lectin (LEA), and the size of the X-mean can reflect the level of polylactosamine residues on the cell surface. Every cell line was also treated only with PE-conjugated streptavidin which served as a control. As shown in Fig. 3A and B, the X-mean of LEA-labeled LS-174T, SW620, SW480 and LoVo cells was 1.169, 1.529, 2.272 and 5.903, respectively. Based on the results, we found that the trend of the intensity of polylactosamine was consistent with the protein expression of $\beta 3 \mathrm{GnT} 8$ in the four colorectal cancer cell lines. That is to say, the expression of polylactosamine in the highly metastatic colorectal cancer cell line LoVo was much stronger than that in the primary colorectal cancer cell line LS-174T. In addition, as shown in Fig. 3C, the glycoprotein affected by polylactosamine had different expression levels in the four colorectal cancer cell lines. With the increased capacity of metastasis, the level of glycoprotein caused by polylactosamine ranged from 49 to $90 \mathrm{kDa}$ and gradually increased, which was in accordance with the flow cytometric analysis. The sizes of these glycoproteins catalyzed by $\beta 3 \mathrm{GnT} 8$ appeared to be related to the metastatic potential of the colorectal cancer cells.

Differential expression of CD147 in the four colorectal cancer cell lines and the effect of $\beta 3 G n T 8$ on glycosylation of CD147 in LS-174T and LoVo cells. Proteins from the four colorectal cancer cells were assessed by western blot analysis. As shown in Fig. 4A, western blot analysis showed that CD147 protein exhibited differential expression in the four colorectal cancer cell lines. The highest level of the CD147 protein was detected in the LoVo cells. The lowest level of the CD147 protein was noted in the LS-174T cells, and its expression levels in the SW480 and SW620 cells were between the values noted in the LoVo and LS-174T cells, which was similar to the
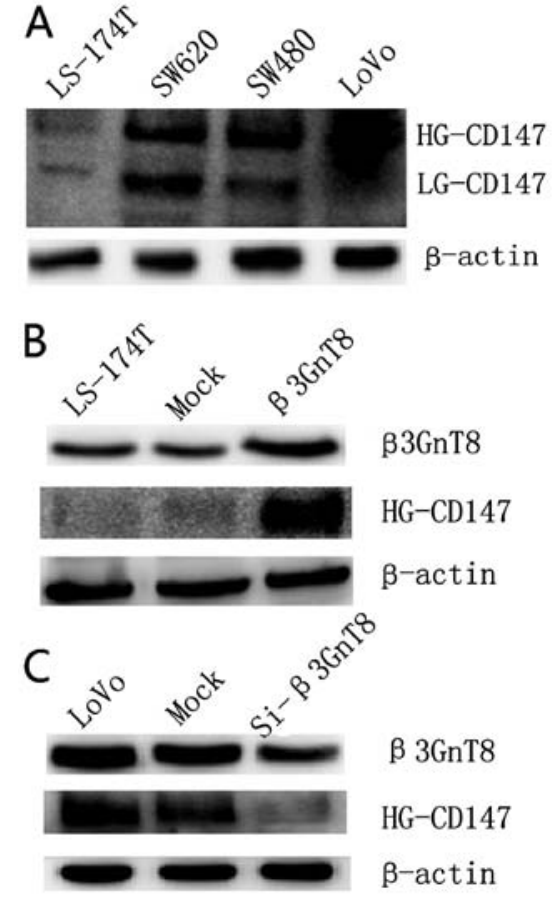

Figure 4. Western blot analysis of CD147 in the four colorectal cancer cell lines and the effect of $\beta 3 \mathrm{GnT} 8$ on the glycosylation of CD147 in LS-174T and LoVo cells. (A) Expression of CD147 in the four colorectal cancer cell lines. (B) $33 \mathrm{GnT} 8$ was upregulated in LS-174T cells, and the level of glycosylation of CD147 was stronger than that in the wild-type and mock cells. (C) $\beta 3 \mathrm{GnT} 8$ was downregulated in LoVo cells, and the level of glycosylation of CD147 was weaker than that in the wild-type and mock cells. In brief, blots were stripped and reprobed with a human $\beta$-actin probe to confirm equal loading, and $30 \mu \mathrm{g}$ of total protein was loaded in each lane. The experiments are representative of three independent experiments with similar results.

trend of $\beta 3 \mathrm{GnT} 8$ in the four colorectal cancer cell lines. To study the effect of $\beta 3 \mathrm{GnT} 8$ on the glycosylation of CD147, we upregulated $\beta 3 \mathrm{GnT} 8$ expression in the LS-174T cells and downregulated $\beta 3 \mathrm{GnT} 8$ in the LoVo cells. The transfected LS-174T and LoVo cells were assessed by western blot 


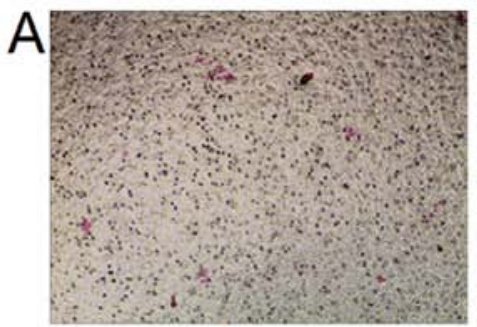

LS-174T

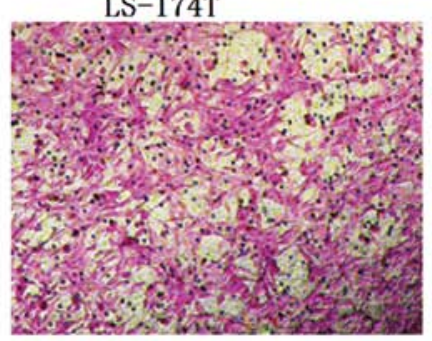

LoVo

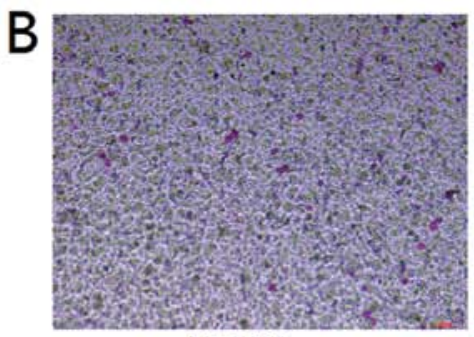

LS-174T

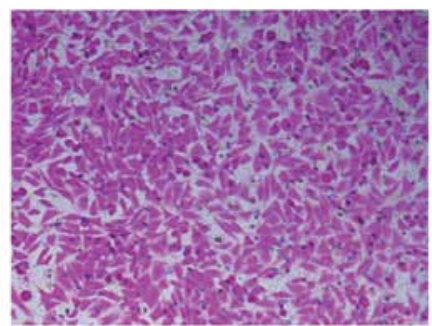

LoVo

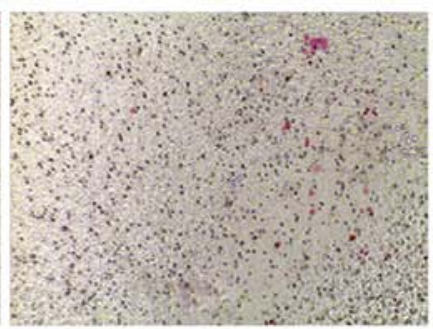

Mock

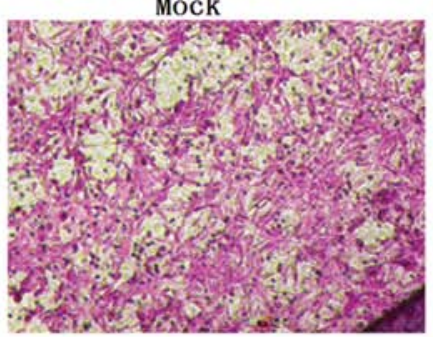

Mock

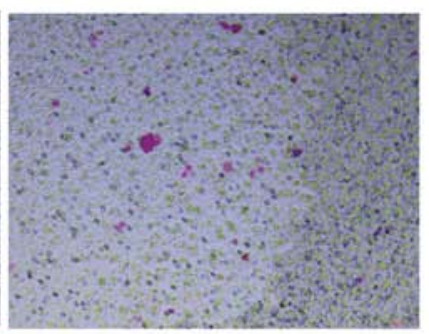

Mock

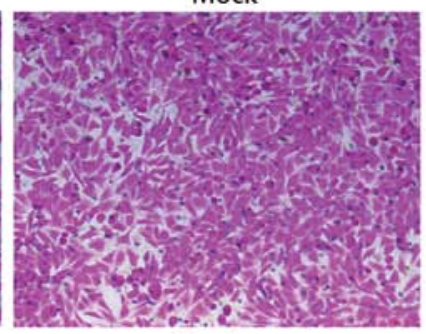

Mock

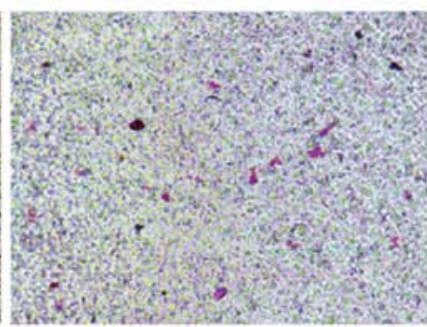

B $3 \mathrm{GnT} 8$

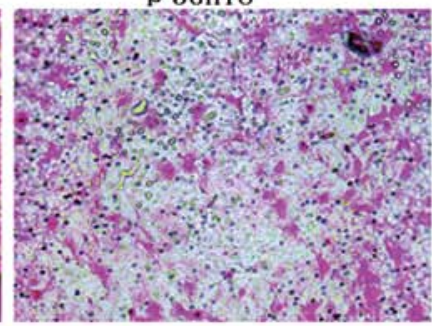

Si- $\beta$ 3GnT8

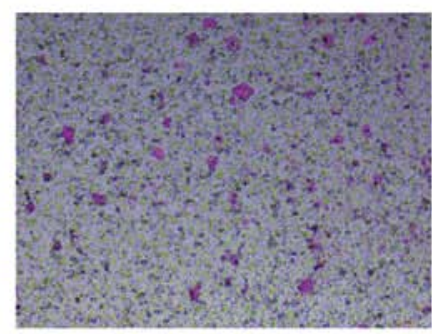

$\beta 3$ GnT8

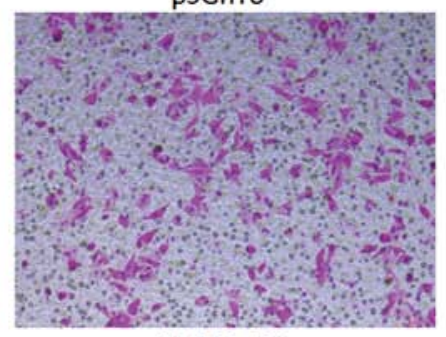

Si- $\beta 3$ GnT8
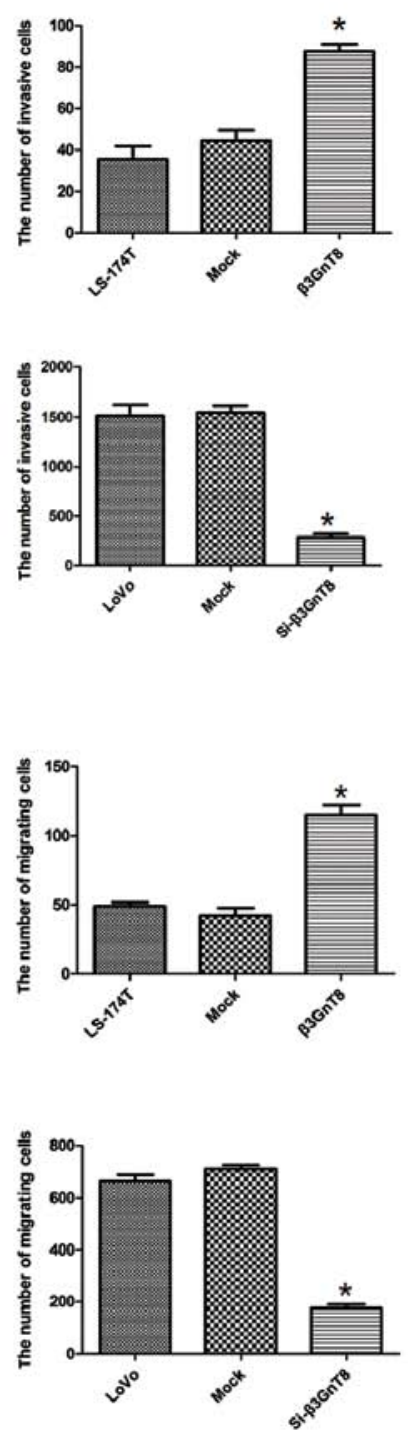

Figure 5. Transwell invasion and migration assays. (A) Transwell invasion assay. Cells were added to the top chambers of 24-well tissue culture plates containing a Matrigel-coated polycarbonate membrane. After incubation for $48 \mathrm{~h}$, the invasive cells were fixed and stained with eosin staining solution and then examined under a light microscope. Representative examples were photographed at x200 magnification. (B) Transwell migration assay. Results are representative of three independent experiments, and bars represent the mean $\pm \mathrm{SD}(\mathrm{P}<0.05$ was considered to indicate a statistically significant result).

analysis. As shown in Fig. 4B and C, HG-CD147 expression was weak in the LS-174T cells and the mock group, while a higher amount of HG-CD147 was observed in the $\beta 3 \mathrm{GnT} 8$ overexpressing cells $(\mathrm{P}<0.05)$, while in the LoVo, mock and Si- $\beta 3$ GnT8 cells, the level of glycosylation of HG-CD147 was reduced apparently with silenced $\beta 3 \mathrm{GnT} 8$ expression when compared to the wild-type and mock group $(\mathrm{P}<0.05)$. The changes in the N-glycosylation level of CD147 indicated that $\beta 3 \mathrm{GnT} 8$ may regulate $\beta 1,6$-branched polylactosamine sugars of CD147.

Effects of $\beta 3 G n T 8$ on the invasive and migratory capabilities of the LS-174T and LoVo cells by Transwell assay. We assessed the effect of $\beta 3 \mathrm{GnT} 8$ on tumor metastasic ability. Transwell migration and invasion chambers coated with Matrigel were used. As shown in Fig. 5B, $\beta 3 \mathrm{GnT}$ 8-overexpressing LS-174T cells exhibited a greater potential to invade and migrate than the wild-type and mock cells $(\mathrm{P}<0.05)$. However, when $\beta 3 \mathrm{GnT} 8$ was downregulated in LoVo cells, the invasive and migratory abilities were markedly inhibited when compared to the wild-type and mock group $(\mathrm{P}<0.05)$. The change in the invasive and migratory abilities of the LS-174T and LoVo cells displayed a similar trend with that of HG-CD147. These results suggest that $\beta 3 \mathrm{GnT} 8$ may regulate metastasis-associated behaviors of colorectal cancer cells through regulation of the glycosylated forms of CD147.

\section{Discussion}

Aberrant glycosylation of cell-surface glycoconjugates is a universal feature of cancer cells. These alterations may be instrumental in the failure of intercellular contact and communication and in the invasive and infiltrative properties 
of cancerous cells (12). Various types of cancer cells, such as U937 (histiocytic lymphoma), ACHN (human kidney glandular cancer), MKN45 (human gastric cancer), A549 (human lung cancer) and Jurkat (acute T-cell leukemia) cells express a large amount of $\mathrm{N}$-glycans having polylactosamine residues as analyzed by HPLC and MS techniques $(13,14)$. Comparison of $\mathrm{N}$-glycans in different colon cancer cells revealed that highly metastatic cancer cells contained more polylactosamine side chains than cells with low metastatic potential (3).

$\beta 3 \mathrm{GnT} 8$ was cloned and characterized by us and by another group $(6,15)$. $\beta 3 \mathrm{GnT} 8$ transfers the GlcNAc sugar to the non-reducing terminus $\beta 1-6$ branched $\mathrm{N}$-glycan to form the Gal/1-4GlcNAc polylactosamine structure in vitro. It is known that glycosyltransferases play an important role in cancer progression mainly by changing sugar chains to alter the function of related glycoproteins. Previous research found that $\beta 1,6$ branched $\mathrm{N}$-linked oligosaccharides are associated with the tumor metastatic potential of gliomas (16). Similar results were also obtained in breast and colorectal cancers (17). The terminal residues of $\beta 1,6$ branched $\mathrm{N}$-oligosaccharides can be substituted by several oligosaccharide chains such as polylactosamine, Le antigen, and 2,3 linked sialic acids $(18,19)$. In addition, $\beta 1,6$ branched $\mathrm{N}$-oligosaccharides are the preferred intermediate for extension with polylactosamine chains (i.e. Gal $\beta 1,4 \mathrm{GlcNAc} \beta 1,3$ repeating units of 2 to $>10$ in length) (19). Therefore, polylactosamine residues on the terminal of $\beta 1,6$ branched $\mathrm{N}$-oligosaccharides which is attributed to the upregulated expression of $\beta 3 \mathrm{GnT} 8$ may play an important role in the progression of colorectal cancer.

It has been reported that highly metastatic colorectal carcinoma cells express more polylactosamine side chains than cells with low metastatic potential (3). To date, few studies concerning the relationship between the polylactosamine residues on $\beta 1,6$ branched $\mathrm{N}$-oligosaccharides and its corresponding glycosyltransferase $\beta 3 \mathrm{GnT} 8$ on the influence of colorectal carcinoma metastasis have been reported.

In the present study, LS-174T, SW620, SW480 and LoVo cell lines were selected because of their differential metastatic properties. LoVo has the highest metastatic potential among the four colorectal cancer cell lines and were derived from a metastatic site, the left supraclavicular region. SW620 cells were derived from a lymph node metastatic site. LS-174T and SW480 cells were derived from the primary site. According to the American Type Culture Collection (ATCC), the patient from whom SW480 was derived later developed metastasis $(20,21)$.

To examine their invasive and migratory abilities, Transwell chamber assays were used. As shown in Fig. 1, the number of LoVo cells that passed through the polycarbonate membrane was higher when compared with that of the LS-174T cells, and the numbers of SW480 and SW620 were between the values noted for the LoVo and LS-174T cells. The results suggest that the invasive and migratory abilities of the four colorectal cancer cell lines were consistent with the metastatic potential. Thus, we examined the expression of $\beta 3 \mathrm{GnT} 8$ which catalyzes the polylactosamine chains on the terminal of $\beta 1,6$ branched $\mathrm{N}$-oligosaccharides in the four colorectal cancer cell lines. Notably, we found that $\beta 3 \mathrm{GnT} 8$ and polylactosamine residues were highest in the LoVo cells and lowest in the LS-174T cells (Figs. 2 and 3). Therefore, the four colorectal cancer cell lines are an appropriate model with which to ascertain whether the expression of $\beta 3 \mathrm{GnT} 8$ correlates with the development of invasion and metastasis in colorectal cancer. To explore the level of polylactosamine residues on the surfaces of the colorectal cancer cells, we used tomato lectin, which can specifically bind to the polylactosamine structure on glycolconjugates (22).

Whether the above biological effects are due to the alteration of $\beta 3 \mathrm{GnT} 8$ and its polylactosamine residues on the $\beta 1,6$ branched $\mathrm{N}$-oligosaccharides requires further study. It has been reported that polylactosamine on $\beta 1,6$ branched $\mathrm{N}$-oligosaccharides plays an important role in facilitating lung-specific metastasis of melanoma B16F1 and B16F10 cells via high affinity galectin-3 (23). The branched $\mathrm{N}$-oligosaccharides formed by $\beta 1,6$ branching were able to modulate the structural and the functional properties of carrier proteins related to cancer metastasis. Some proteins carrying the types of oligosaccharides have been shown to express cell adhesion molecules (CAMs) such as $\beta 1$ integrin, CD44 and cadherins $(18,24)$.

A previous study showed that CD147 is a major carrier of $\beta 1$,6-branched polylactosamine sugars in HT1080 tumor cells (25), and previous studies confirmed that only HG-CD147 plays an important role in the induction of MMPs, thereby leading to extracellular matrix degradation and increased tumor growth and metastasis $(11,26)$. Moreover, in breast cancer cells and in nude mice, it can markedly increased tumor growth and metastasis $(27,28)$. In addition, HG-CD147 was found to contribute to lymphatic metastasis potential in mouse hepatocarcinoma cells by altering the level of N-glycans (29). Huang et al (30) confirmed that $\mathrm{N}$-glycosylation of CD147, particularly $\beta 1,6$ branched N-glycans contributes to MMP-inducing activity in SMMC-7721 cells. Hence, we speculate that HG-CD147 as a major carrier of $\beta 1,6$-branched polylactosamine sugars on $\mathrm{N}$-glycans may be involved in the metastatic potential of colorectal cancer. In the present study, we found that the trend of the level of HG-CD147 was similar to the invasive potential of the four colorectal cancer cells (Fig. 4A), and HG-CD147 was strongly expressed on LoVo cells with high invasive potential while its expression was low on LS-174T cells with low invasive potential. In addition, by introducing exogenous $\beta 3$ GnT 8 into LS-174T cells, the HG-CD147 expression markedly increased, while LG-CD147 expression was not detected in the LS-174T cells (data not shown). Perhaps, CD147 in LS-174T cells was transformed from LG-CD147 to HG-CD147. Downregulation of $\beta 3 \mathrm{GnT} 8$ in LoVo cells resulted in obviously decreased HG-CD147 (Fig. 4B and C). Moreover, in the present study, exogenous $\beta 3 \mathrm{GnT} 8$ caused a dramatic alteration in the glycosylated forms of CD147. Furthermore, after exogenous $\beta 3 \mathrm{GnT} 8 \mathrm{cDNA}$ was transfected into LS-174T cells, a dramatic increase in the invasive and migratory potentials were noted. In contrast, when $\beta 3 \mathrm{GnT} 8$ expression in LoVo cells was downregulated, the invasive and migratory abilities were inhibited (Fig. 5). The results suggest that $\beta 3 \mathrm{GnT} 8$ regulates the metastasis-associated behaviors of colorectal cancer cells by altering the glycosylated forms of CD147.

In summary, the present study demonstrated that the expression of $\beta 3 \mathrm{GnT} 8$ and polylactosamine residues on $\beta 1,6$ branched $\mathrm{N}$-oligosaccharides is associated with the metastasic potential of colorectal cancer cells. They may promote the invasive and 
migratory abilities by modulating the $\mathrm{N}$-glycosylated forms of CD147. This property may provide a valuable strategy for the diagnosis and prognosis of colorectal cancer.

\section{Acknowledgements}

The present study was supported by the National Natural Science Foundation of China (no. 31170772) and the Suzhou Municipal Natural Science Foundation (SY201208).

\section{References}

1. Demetriou M, Granovsky M, Quaggin S and Dennis JW: Negative regulation of T-cell activation and autoimmunity by Mgat5 N-glycosylation. Nature 409: 733-739, 2001.

2. Dennis JW, Granovsky M and Warren CE: Glycoprotein glycosylation and cancer progression. Biochim Biophys Acta 1473: 21-34, 1999.

3. Saitoh O, Wang WC, Lotan R and Fukuda M: Differential glycosylation and cell surface expression of lysosomal membrane glycoproteins in sublines of a human colon cancer exhibiting distinct metastatic potentials. J Biol Chem 267 5700-5711, 1992.

4. Figer A, Perez-Staub N, Carola E, et al: FOLFOX in patients aged between 76 and 80 years with metastatic colorectal cancer: an exploratory cohort of the OPTIMOX1 study. Cancer 110: 2666-2671, 2007.

5. Mansouri D, McMillan DC, Crighton EM and Horgan PG: Screening for colorectal cancer: what is the impact on the determinants of outcome? Crit Rev Oncol Hematol 85: 342-349, 2013.

6. Ishida $\mathrm{H}$, Togayachi A, Sakai $\mathrm{T}$, et al: A novel $\beta 1,3-\mathrm{N}$-acetylglucosaminyltransferase $(\beta 3 \mathrm{Gn}-\mathrm{T} 8)$, which synthesizes poly-N-acetyllactosamine, is dramatically upregulated in colon cancer. FEBS Lett 579: 71-78, 2005

7. Shen L, Liu Z, Tu Y, Xu L, Sun X and Wu S: Regulation of MMP-2 expression and activity by $\beta-1,3-\mathrm{N}$-acetylglucosaminyltransferase-8 in AGS gastric cancer cells. Mol Biol Rep 38: 1541-1550, 2011

8. Polette M, Gilles C, Marchand V, et al: Tumor collagenase stimulatory factor (TCSF) expression and localization in human lung and breast cancers. J Histochem Cytochem 45: 703-709, 1997.

9. Ellis SM, Nabeshima K and Biswas C: Monoclonal antibody preparation and purification of a tumor cell collagenase-stimulatory factor. Cancer Res 49: 3385-3391, 1989.

10. Yu XL, Jiang JL, Li L, Feng Q, Xu J and Chen ZN: The glycosylation characteristic of hepatoma-associated antigen HAb18G/ CD147 in human hepatoma cells. Int J Biochem Cell Biol 38: 1939-1945, 2006.

11. Sun J and Hemler ME: Regulation of MMP-1 and MMP-2 production through CD147/extracellular matrix metalloproteinase inducer interactions. Cancer Res 61: 2276-2281, 2001

12. Carvalho FC, Soares SG, Tamarozzi MB, Rego EM and RoqueBarreira MC: The recognition of N-glycans by the lectin ArtinM mediates cell death of a human myeloid leukemia cell line. PLoS One 6: e27892, 2011.

13. Naka R, Kamoda S, Ishizuka A, Kinoshita M and Kakehi K: Analysis of total N-glycans in cell membrane fractions of cancer cells using a combination of serotonin affinity chromatography and normal phase chromatography. J Proteome Res 5: 88-97, 2006.
14. Mitsui Y, Yamada K, Hara S, Kinoshita M, Hayakawa T and Kakehi K: Comparative studies on glycoproteins expressing polylactosamine-type $\mathrm{N}$-glycans in cancer cells. J Pharm Biomed Anal 70: 718-726, 2012.

15. Huang C, Zhou J, Wu S, Shan Y, Teng S and Yu L: Cloning and tissue distribution of the human B3GALT7 gene, a member of the beta1,3-glycosyltransferase family. Glycoconj J 21: 267-273, 2004.

16. Yamamoto H, Swoger J, Greene S, et al: $\beta 1,6-\mathrm{N}$-acetylglucosamine-bearing N-glycans in human gliomas: implications for a role in regulating invasivity. Cancer Res 60: 134-142, 2000.

17. Fernandes B, Sagman U, Auger M, Demetrio M and Dennis JW: $\beta 1-6$ branched oligosaccharides as a marker of tumor progression in human breast and colon neoplasia. Cancer Res 51: 718-723, 1991.

18. Hakomori S: Tumor malignancy defined by aberrant glycosylation and sphingo(glyco)lipid metabolism. Cancer Res 56: 5309-5318, 1996.

19. van den Eijnden DH, Koenderman AH and Schiphorst WE: Biosynthesis of blood group i-active polylactosaminoglycans. Partial purification and properties of an UDP-GlcNAc: $N$ acetyllactosaminide $\beta 1 \rightarrow 3-N$-acetylglucosaminyltransferase from Novikoff tumor cell ascites fluid. J Biol Chem 263: 12461-12471, 1988

20. Flatmark K, Maelandsmo GM, Martinsen M, Rasmussen $H$ and Fodstad O: Twelve colorectal cancer cell lines exhibit highly variable growth and metastatic capacities in an orthotopic model in nude mice. Eur J Cancer 40: 1593-1598, 2004.

21. Kusakai G, Suzuki A, Ogura T, Kaminishi M and Esumi H: Strong association of ARK5 with tumor invasion and metastasis. J Exp Clin Cancer Res 23: 263-268, 2004.

22. Seko A and Yamashita K: Activation of $\beta 1,3-\mathrm{N}$-acetylglucosaminyltransferase-2 (beta $3 \mathrm{Gn}-\mathrm{T} 2$ ) by $\beta 3 \mathrm{Gn}-\mathrm{T} 8$. Possible involvement of $\beta 3 \mathrm{Gn}-\mathrm{T} 8$ in increasing poly-N-acetyllactosamine chains in differentiated HL-60 cells. J Biol Chem 283: 33094-33100, 2008.

23. Krishnan V, Bane SM, Kawle PD, Naresh KN and Kalraiya RD: Altered melanoma cell surface glycosylation mediates organ specific adhesion and metastasis via lectin receptors on the lung vascular endothelium. Clin Exp Metastasis 22: 11-24, 2005.

24. Demetriou M, Nabi IR, Coppolino M, Dedhar S and Dennis JW: Reduced contact-inhibition and substratum adhesion in epithelial cells expressing GlcNAc-transferase V. J Cell Biol 130: 383-392, 1995.

25. Tang W, Chang SB and Hemler ME: Links between CD147 function, glycosylation, and caveolin-1. Mol Biol Cell 15: 4043-4050, 2004.

26. Guo H, Zucker S, Gordon MK, Toole BP and Biswas C: Stimulation of matrix metalloproteinase production by recombinant extracellular matrix metalloproteinase inducer from transfected Chinese hamster ovary cells. J Biol Chem 272: 24-27, 1997.

27. Zucker S, Hymowitz M, Rollo EE, et al: Tumorigenic potential of extracellular matrix metalloproteinase inducer. Am J Pathol 158: 1921-1928, 2001

28. Caudroy S, Polette M, Nawrocki-Raby B, et al: EMMPRINmediated MMP regulation in tumor and endothelial cells. Clin Exp Metastasis 19: 697-702, 2002.

29. Fan J, Wang S, Yu S, He J, Zheng W and Zhang J: N-acetylglucosaminyltransferase IVa regulates metastatic potential of mouse hepatocarcinoma cells through glycosylation of CD147. Glycoconj J 29: 323-334, 2012.

30. Huang W, Luo WJ, Zhu P, et al: Modulation of CD147-induced matrix metalloproteinase activity: role of CD147 N-glycosylation. Biochem J 449: 437-448, 2013. 